\title{
Clinical Significance of an Increased Cochlear 3D Fluid- Attenuated Inversion Recovery Signal Intensity on an MR Imaging Examination in Patients with Acoustic Neuroma
}

\author{
D.Y. Kim, J.H. Lee, M.J. Goh, Y.S. Sung, Y.J. Choi, R.G. Yoon, S.H. Cho, J.H. Ahn, H.J. Park, and J.H. Baek
}

\begin{abstract}
BACKGROUND AND PURPOSE: The increased cochlear signal on FLAIR images in patients with acoustic neuroma is explained by an increased concentration of protein in the perilymphatic space. However, there is still debate whether there is a correlation between the increased cochlear FLAIR signal and the degree of hearing disturbance in patients with acoustic neuroma. Our aim was to investigate the clinical significance of an increased cochlear 3D FLAIR signal in patients with acoustic neuroma according to acoustic neuroma extent in a large patient cohort.
\end{abstract}

MATERIALS AND METHODS: This retrospective study enrolled 102 patients with acoustic neuroma, who were divided into 2 groups based on tumor location; 22 tumors were confined to the internal auditory canal and 80 extended to the cerebellopontine angle cistern. Pure tone audiometry results and hearing symptoms were obtained from medical records. The relative signal intensity of the entire cochlea to the corresponding brain stem was calculated by placing regions of interest on 3D FLAIR images. Statistical analysis was performed to compare the cochlear relative signal intensity between the internal auditory canal acoustic neuroma and the cerebellopontine angle acoustic neuroma. The correlation between the cochlear relative signal intensity and the presence of hearing symptoms or the pure tone audiometry results was investigated.

RESULTS: The internal auditory canal acoustic neuroma cochlea had a significantly lower relative signal intensity than the cerebellopontine angle acoustic neuroma cochlea $(0.42 \pm 0.15$ versus $0.60 \pm 0.17, P<.001)$. The relative signal intensity correlated with the audiometric findings in patients with internal auditory canal acoustic neuroma $(r=0.471, P=.027)$ but not in patients with cerebellopontine angle acoustic neuroma $(P=.427)$. Neither internal auditory canal acoustic neuroma nor cerebellopontine angle acoustic neuroma showed significant relative signal intensity differences, regardless of the presence of hearing symptoms $(P>.5)$.

CONCLUSIONS: The cochlear signal on FLAIR images may be an additional parameter to use when monitoring the degree of functional impairment during follow-up of patients with small acoustic neuromas confined to the internal auditory canals.

ABBREVIATIONS: $\mathrm{AN}=$ acoustic neuroma; $\mathrm{CPA}=$ cerebellopontine angle; $\mathrm{IAC}=$ internal auditory canal; $\mathrm{PTA}=$ pure tone audiometry; $\mathrm{rSI}=$ relative signal intensity

$\mathbf{T}$

he fluid of the inner ear is normally suppressed on 3D fluidattenuated inversion recovery MR images. Increased signal intensity of the fluid on FLAIR MR images has been reported in various diseases, including sudden sensorineural hearing loss,

Received December 31, 2013; accepted after revision February 17, 2014

From the Departments of Radiology and Research Institute of Radiology (D.Y.K., J.H.L., M.J.G., Y.S.S., Y.J.C., R.G.Y., S.H.C., J.H.B.) and Otorhinolaryngology-Head and Neck Surgery (J.H.A., H.J.P.), University of Ulsan College of Medicine, Asan Medical Center, Seoul, Republic of Korea.

Paper previously presented at: Annual Meeting of the Radiological Society of North America, December 1-6, 2013; Chicago, Illinois.

Please address correspondence to Jeong Hyun Lee, MD, PhD, Department of Radiology and Research Institute of Radiology, University of Ulsan College of Medi-

cine, Asan Medical Center, 86 Asanbyeongwon-Gil, Songpa-Gu, Seoul 138-736, Republic of Korea; e-mail: jeonghlee@amc.seoul.kr

http://dx.doi.org/10.3174/ajnr.A3936 labyrinthine hemorrhage, otosclerosis, Ramsay Hunt syndrome, and acoustic neuromas (ANs). ${ }^{1-6}$ The increased cochlear signal on FLAIR images in patients with ANs is explained by an increased concentration of protein in the perilymphatic space. ${ }^{7-12}$

FLAIR MR imaging is sensitive to fluids with a high protein content. ${ }^{13-17}$ Furthermore, 3D-FLAIR imaging can minimize the undesired inflow artifacts of CSF flow, has a higher signal-tonoise ratio and spatial resolution, and allows recognition of subtle compositional changes of the inner ear fluid. ${ }^{18-21}$ Therefore, one can assume that the increased protein content in the cochlear perilymph of patients with ANs can be detected on 3D FLAIR imaging with a high sensitivity and good spatial resolution.

Several researchers recently investigated whether there was a correlation between the increased cochlear FLAIR signal in patients with ANs and the degree of their hearing disturbance. ${ }^{2,5}$ 


\begin{tabular}{|c|c|c|c|}
\hline & \multirow[b]{2}{*}{ Total } & \multicolumn{2}{|c|}{ Tumor Location } \\
\hline & & $\operatorname{IAC}\left(\mathrm{AN}_{\mathrm{IAC}}\right)$ & $\begin{array}{c}\text { IAC and CPA } \\
\left.\text { Cistern (AN }{ }_{\text {CPA }}\right)\end{array}$ \\
\hline No. of patients & 102 & 22 & 80 \\
\hline Male/female & $58: 44$ & $10: 12$ & $48: 32$ \\
\hline Mean age (yr) & $49.9 \pm 12.4^{\mathrm{a}}$ & $50.1 \pm 12.6^{\mathrm{a}}$ & $49.8 \pm 12.4^{\mathrm{a}}$ \\
\hline Time interval between PTA and MRI (days) & $25.7 \pm 31.2^{\mathrm{a}}$ & $23.1 \pm 28.1^{\mathrm{a}}$ & $26.4 \pm 32.0^{\mathrm{a}}$ \\
\hline No. of patients with hearing disturbance & $70(69 \%)$ & $11(50 \%)$ & $59(74 \%)$ \\
\hline No. of patients with tinnitus & $45(44 \%)$ & $9(41 \%)$ & $36(45 \%)$ \\
\hline
\end{tabular}

${ }^{\mathrm{a}}$ Mean $\pm \mathrm{SD}$.

However, no such correlation was found, nor was there any difference in the cochlear FLAIR signal according to AN extent.

Accordingly, the purpose of this study was to investigate the clinical significance of an increased cochlear 3D FLAIR signal in a large number patients with ANs by correlating imaging results with audiometric findings and hearing symptoms according to the extent of ANs after dividing the auditory neuromas into 2 groups: those confined to the internal auditory canal $\left(\mathrm{AN}_{\mathrm{IAC}}\right)$ and those extending into the cerebellopontine cistern $\left(\mathrm{AN}_{\mathrm{CPA}}\right)$.

\section{MATERIALS AND METHODS}

\section{Study Participants}

This retrospective study was approved by the institutional review board of our hospital.

Between 2008 and 2012, one hundred twenty-two patients with $\mathrm{AN}$ who had been admitted to our hospital were identified according to the radiology report data base. Twenty patients were excluded for one of the following reasons: 1) no pretreatment 3D FLAIR MR imaging or pure tone audiometry (PTA) data $(n=$ $13)$; 2) any other causes of hearing problems such as chronic otomastoiditis $(n=6)$ based on conventional T1- and T2-weighted images; or 3) intralabyrinthine schwannoma $(n=1)$. Finally, 102 patients were enrolled in the study, including 64 patients with pathologic confirmation of AN after surgery and 38 patients diagnosed according to the typical MR imaging appearance of AN and who underwent gamma knife radiosurgery. ANs were divided into 2 groups: those confined to the internal auditory canal (22 cases) and those involving the cerebellopontine angle cistern and the internal auditory canal (IAC) (80 cases) (Table 1). The results of the preoperative PTA - conducted within 3 months of the pretreatment MR imaging-were obtained (mean intervals: $23.1 \pm 28.1$ days for $\mathrm{AN}_{\mathrm{IAC}} ; 26.4 \pm 32.0$ days for $\mathrm{AN}_{\mathrm{CPA}}$ ). We also investigated whether patients had hearing symptoms such as a hearing disturbance or tinnitus according to a review of the electronic medical records.

\section{MR Imaging}

All MR imaging examinations, including coronal T1- and T2weighted, turbo spin-echo, and axial 3D T2 and FLAIR imaging, were performed on a 3T MR imaging unit (Achieva; Philips Healthcare, Best, the Netherlands) with an 8-channel head coil. We used a variable refocusing flip angle technique to obtain isotropic 3D T2-weighted and FLAIR MR images. Axial 3D FLAIR MR imaging was performed with the following parameters: $\mathrm{TR}=$ $8000 \mathrm{~ms} ; \mathrm{TE}_{\mathrm{eff}}=280 \mathrm{~ms}$; inversion time $=2400 \mathrm{~ms}$; fat saturation $=$ spectral adiabatic inversion recovery; flip angle $=90^{\circ}$; number of signal averages $=1$; echo-train length $=80$; number of encoding steps $=300 ; \mathrm{FOV}=180 \times 180 \mathrm{~mm}$; matrix $=300 \times$
300 (reconstruction matrix $=512 \times 512$ ); voxel size $=0.6 \times 0.6 \times 0.6 \mathrm{~mm}^{3}$; slab number $=1$; slab thickness $=40 \mathrm{~mm}$; section thickness $=1.2 \mathrm{~mm}$; spacing between sections $=0.6 \mathrm{~mm}$; acquisition time $=8$ minutes 48 seconds; and sensitivity encoding factor $=2$.

Axial 3D T2-weighted imaging was performed with the following parameters: $\mathrm{TR}=2000 \mathrm{~ms} ; \mathrm{TE}_{\mathrm{eff}}=250 \mathrm{~ms}$; flip angle $=90^{\circ}$; number of signal averages $=2$; echo-train length $=70$; number of encoding steps $=300$; FOV $=$ $180 \times 180 \mathrm{~mm}$; matrix $=300 \times 300$ (reconstruction matrix $=$ $512 \times 512)$; voxel size $=0.6 \times 0.6 \times 0.6 \mathrm{~mm}^{3}$; slab number $=1$; slab thickness $=40 \mathrm{~mm}$; section thickness $=1.2 \mathrm{~mm}$; spacing between sections $=0.6 \mathrm{~mm}$; acquisition time $=4$ minutes 58 seconds; and sensitivity encoding factor $=2$.

\section{Image Analysis}

Image analysis was performed with ImageJ, a public domain, Java-based image processing program developed at the National Institutes of Health (Bethesda, Maryland; http://rsbweb.nih.gov/ ij/). Axial 3D FLAIR and conventional T2-weighted images were reviewed to determine the presence of any coexisting abnormalities in the pons, where a reference region-of-interest was to be placed. Our review revealed no patient with a pontine signalintensity abnormality. After importing the DICOM files of the axial 3D FLAIR and 3D T2-weighted MR images into ImageJ, a neuroradiologist with 5 years of clinical experience placed ROIs on the cochlea on the affected side of the AN and the corresponding brain stem (Fig 1). ROIs were placed directly on the entire cochlea on each section of the 3D FLAIR images by using the Wand tool of ImageJ, which allowed reliable definition of the boundary of the cochlea with increased signal intensity. When the cochlear signal intensity was insufficient to define the boundary, the region-of-interest placements were checked by reference to 3D T2 MR images at the same level. Circular ROIs of the same size (20 pixels, $7.2 \mathrm{~mm}^{2}$ ) were carefully placed at the center of the brain stem on the same level as every measured cochlea section. We obtained the mean value of the signal intensities within the ROIs of the cochlea and the brain stem, respectively, and calculated the relative signal intensity (rSI) of the entire cochlea to the brain stem. Another neuroradiologist with 4 years of clinical experience independently measured the signal intensities of the cochlea and the brain stem in the same way. These results were compared to determine the interobserver agreement.

\section{Pure Tone Audiometry}

Hearing levels were evaluated with an audiometer (Orbiter922; Madsen, Taastrup, Denmark) in a sound-insulated chamber. The average hearing levels of bone and air conduction were calculated in ANs as the mean of the hearing levels measured at 250, 500, 1000, 2000, and $4000 \mathrm{~Hz}$ based on the American Academy of Otolaryngology Head and Neck Surgery guidelines for AN. ${ }^{22}$ 

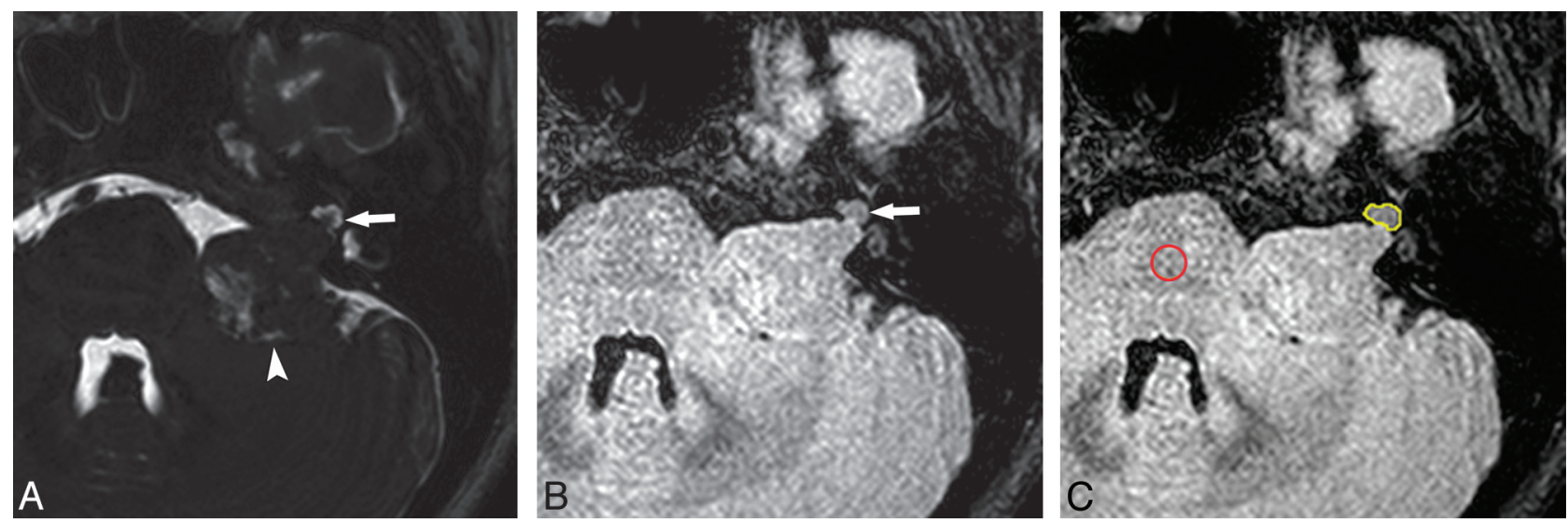

FIG 1. $A N_{C P A}$ with markedly increased cochlear signal intensity in a 71-year-old man. A, On 3D T2-weighted image $(T R=2000 \mathrm{~ms}$, TE $=250 \mathrm{~ms}$, flip angle $=90^{\circ}$ ), a large acoustic neuroma (arrowhead) is visible in the widened internal auditory canal and the cerebellopontine angle cistern that compresses the brain stem. High signal intensity in the cochlea (arrow) is also observed. B, On 3D FLAIR image (TR $=8000 \mathrm{~ms}$, TE $=280 \mathrm{~ms}$, inversion time $=2400 \mathrm{~ms}$, flip angle $=90^{\circ}$ ), the signal intensity of the affected cochlea is markedly increased (arrow) in contrast to the suppressed CSF signal. C, To measure the signal intensity of the cochlea, a region inside the cochlea was selected by using the Wand tool, which resulted in automatic growing of the region of interest by comparing the signal intensities of all unallocated neighboring pixels with that of the selected region with a predetermined threshold (yellow circle). A circular region of interest (red circle) was placed on the 3D FLAIR image to measure the signal intensity of the pons. These were repeated for each section showing the cochlear turns. The relative signal intensity of the cochlea is 0.690 . The PTA result of this patient was a $42-\mathrm{dB}$ hearing level.
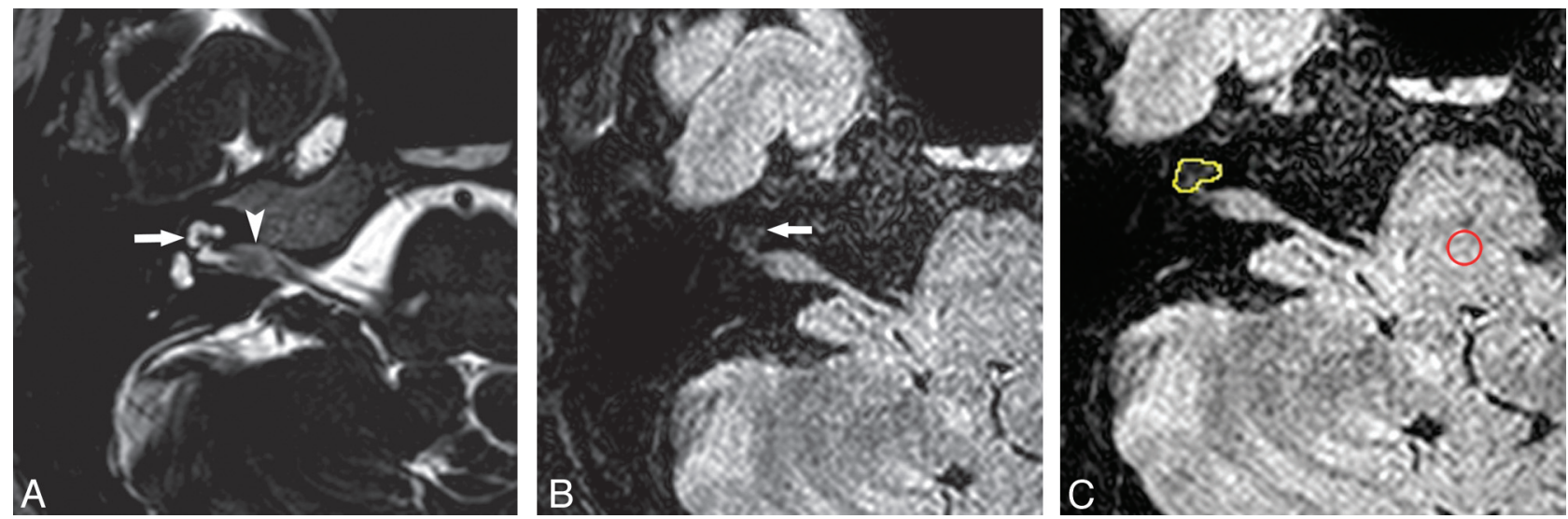

FIG 2. $\mathrm{AN}_{\mathrm{IAC}}$ with a slightly increased cochlear signal intensity in a 39-year-old woman. $A$, On $3 \mathrm{D}$ T2-weighted image $(\mathrm{TR}=2000 \mathrm{~ms}$, TE $=250 \mathrm{~ms}$, flip angle $=90^{\circ}$ ), there is a small acoustic neuroma (arrowhead) that is confined to the internal auditory canal. High signal intensity of the cochlear fluid (arrow) is also observed. B, On 3D FLAIR image (TR $=8000 \mathrm{~ms}$, TE $=280 \mathrm{~ms}$, inversion time $=2400 \mathrm{~ms}$, flip angle $\left.=90^{\circ}\right)$, the signal intensity of the affected cochlea is slightly increased (arrow). C, Two ROIs are placed on the 3D FLAIR image to measure the signal intensities of the cochlea (yellow circle) and the pons (red circle) in the same way as described in Fig 1. The relative signal intensity of the cochlea is calculated as 0.294 from the region-of-interest measurement. The PTA result of this patient was an 11-dB hearing level.

\section{Statistical Analysis}

Independent $t$ tests were performed to compare the rSIs of the cochlea and the PTA results between the $\mathrm{AN}_{\mathrm{IAC}}$ and $\mathrm{AN}_{\mathrm{CPA}}$ groups. Pearson correlation tests for $\mathrm{AN}_{\mathrm{ALL}}, \mathrm{AN}_{\mathrm{CPA}}$, and $\mathrm{AN}_{\mathrm{IAC}}$ were used to investigate the correlation between the PTA and rSI results. Independent $t$ tests for $\mathrm{AN}_{\mathrm{CPA}}$ and Mann-Whitney $U$ tests for $\mathrm{AN}_{\mathrm{IAC}}$ were used to compare the rSIs according to the existence of hearing symptoms. All statistical analyses were performed with the Statistical Package for the Social Sciences (Version 18 for Windows; IBM, Armonk, New York). Interobserver agreement for the rSI of the entire cochlea to the brain stem between 2 observers was investigated by using an intraclass correlation coefficient.

\section{RESULTS}

Table 1 summarizes the clinical findings, including the demographic data and the frequency of hearing disturbance or tinnitus, obtained in this study. Statistical analysis revealed no significant differences in the age, sex, or time interval from PTA to MR imaging examination between those patients with $\mathrm{AN}_{\mathrm{IAC}}$ and those with $\mathrm{AN}_{\mathrm{CPA}}$. Figures 1 and 2 show representative $\mathrm{AN}_{\mathrm{CPA}}$ and $\mathrm{AN}_{\mathrm{IAC}}$ cases.

The PTA results and the mean values of the cochlear rSIs in the 3D FLAIR MR images are summarized in Table 2. The PTA results demonstrated that hearing ability was significantly more impaired in patients with $\mathrm{AN}_{\mathrm{CPA}}$ than in those with $\mathrm{AN}_{\mathrm{IAC}}(46.2 \pm 21.4 \mathrm{~dB}$ versus $30.3 \pm 17.3 \mathrm{~dB} ; P<.001)$. The mean $\mathrm{rSI}$ was also significantly higher in patients with $\mathrm{AN}_{\mathrm{CPA}}$ than in those with $\mathrm{AN}_{\mathrm{IAC}}(0.60 \pm 0.17$ versus $0.42 \pm 0.15 ; P<.001)$. The correlation test revealed that the hearing impairment measured by PTA and the rSI of the cochlea on 3D FLAIR MR images was moderately correlated in patients with $\operatorname{AN}_{\text {IAC }}(r=0.471 ; P=.027)$ and weakly correlated in all patients $(r=$ $0.277 ; P=.005)$. There was no significant correlation between the hearing impairment measured by PTA and the cochlear rSI on 3D 
Table 2: Pure tone audiometry and rSI results of the cochlea on 3D FLAIR MR images according to tumor location

\begin{tabular}{lcccc}
\hline & & \multicolumn{2}{c}{ Tumor Location } & \\
\cline { 3 - 4 } & & & IAC and CPA & \\
& Total & IAC (AN $_{\text {IAC }}$ ) & Cistern (AN CPA $)$ & $P^{\text {a }}$ \\
\hline Measured cochlear volume $\left(\mathrm{mm}^{3}\right)$ & $181.98 \pm 26.5$ & $177.5 \pm 26.0$ & $183.1 \pm 25.8$ & .637 \\
PTA (dB) & $43.4 \pm 21.5$ & $30.3 \pm 17.3$ & $46.2 \pm 21.4$ & $<.001$ \\
rSI of the cochlea on 3D FLAIR $^{\mathrm{b}}$ & $0.57 \pm 0.18$ & $0.42 \pm 0.15$ & $0.60 \pm 0.17$ & $<.001$ \\
Correlation coefficient $^{\mathrm{c}}$ & $r=0.277$ & $r=0.471$ & $r=0.090$ & \\
$P^{\mathrm{c}}$ & .005 & .027 & .427 & \\
\hline
\end{tabular}

Note:- rSI indicates relative signal intensity divided by the mean of the signal intensities of the corresponding brain stem.

a $P$ between the results of $A N_{I A C}$ and $A N_{C P A}$

${ }^{\mathrm{b}}$ The results are expressed as the mean \pm SD.

${ }^{c}$ Correlation coefficient and $P$ between the results of PTA and the rSI of the cochlea on 3D FLAIR images. may be due to the methods used to measure the cochlear signal. Two of these previous studies used small ROIs at the basal/middle turn (ROIs ranging from 2 to $3 \mathrm{~mm}^{2}$ ) or at the most hyperintense area (ROIs ranging from 1.2 to $1.5 \mathrm{~mm}^{2}$ ) of the cochlea, ${ }^{1,2}$ thus resulting in higher mean values such as $0.89 \pm 0.18$ and $0.99 \pm 0.29$, respectively. In the study of Yamazaki et al, ${ }^{5}$ the authors drew a region of interest encompassing the 3 turns of each cochlea at the level of the modiolus on a single image, and the result was $0.59 \pm 0.18$, which is similar to ours. In contrast to these previous studies, we mea-
Table 3: Cochlear rSI results as determined by the presence of symptoms in patients with $A N_{I A C}$ and $A N_{\text {CPA }}$

\begin{tabular}{lcc} 
& \multicolumn{2}{c}{ Cochlear rSls } \\
\cline { 2 - 3 } Symptoms & $\mathrm{AN}_{\text {IAC }}$ & AN $_{\text {CPA }}$ \\
\hline Hearing disturbance & & \\
No $^{\mathrm{a}}$ & $0.418 \pm 0.150$ & $0.609 \pm 0.164$ \\
Yes $^{\mathrm{a}}$ & $0.419 \pm 0.127$ & $0.615 \pm 0.185$ \\
$P^{\mathrm{b}}$ & .870 & .600 \\
Tinnitus $^{\mathrm{N}}$ & & \\
No $^{\mathrm{a}}$ & $0.388 \pm 0.139$ & $0.590 \pm 0.139$ \\
Yes $^{\mathrm{a}}$ & $0.464 \pm 0.124$ & $0.628 \pm 0.189$ \\
$P^{\mathrm{b}}$ & .443 & .506 \\
\hline
\end{tabular}

a The results are expressed as mean \pm SD.

${ }^{\mathrm{b}} \mathrm{P}$ according to the presence of symptoms in patients with $\mathrm{AN}_{\mathrm{IAC}}$ and $A N_{C P A}$.

FLAIR MR images in patients with $\mathrm{AN}_{\mathrm{CPA}}(r=0.090 ; P=.427)$. There was no significant difference in the rSI of the cochlea on $3 \mathrm{D}$ FLAIR images according to the presence of subjective hearing symptoms, such as hearing disturbance or tinnitus, in patients with $\mathrm{AN}_{\mathrm{IAC}}$ and $\mathrm{AN}_{\mathrm{CPA}}$ (Table 3).

Interobserver agreement for the rSI of the entire cochlea to the pons between 2 observers was excellent, with an intraclass correlation coefficient score of $0.928(P<.001)$.

\section{DISCUSSION}

Our study results can be summarized as follows: 1) The increased cochlear signal of intracanalicular ANs on 3D FLAIR images correlated with the degree of hearing impairment measured by PTA; and 2) the degree of correlation was stronger with tumors confined to the IAC compared with the result of all patients, though there was no correlation with tumors extending to the CPA cistern. In addition, the cochlear signal intensity on 3D FLAIR images was significantly higher in patients with $\mathrm{AN}_{\mathrm{CPA}}$ than in those with $\mathrm{AN}_{\mathrm{IAC}}$.

Previous studies have reported results that contrast with those of our present study. They found no correlation between the audiometric results and the degree of the signal increase of the cochlea ${ }^{2,5}$ or no difference in the rSI of the cochlea on FLAIR images according to tumor extent. ${ }^{1,2,5}$

Our study has several advantages over previous studies. First, we measured the average signal intensity of the entire cochlea, including all 3 turns on each section of the MR images. In our analyses, the mean rSI of the affected cochlea on 3D FLAIR images was $0.57 \pm 0.18$ in all patients. A literature review revealed 3 articles that reported increased rSIs of the cochlea on FLAIR images in patients with ANs, ranging from 0.59 to $0.99 .^{1,2,5}$ The wide range in the reported values sured the average signal intensity of the entire cochlea in our current study, including all 3 turns on each section of the MR images, to obtain the most representative value of the cochlear signal change. The reliability of our method is also supported by the almost perfect interobserver agreement for the rSI of the entire cochlea to the brain stem. This interobserver reliability probably results from the objectivity of the analytic method, which used the seeded region-growing method with a constant threshold for the ROIs to determine the boundary between the small cochlea and the adjacent petrous bone. Another advantage of our study is that it contains the largest series of any of the studies to date investigating the correlation between increased cochlear FLAIR signal and the audiometric results in patients with ANs. We, therefore, determined that the degree of signal increase of the cochlea on 3D FLAIR MR images is significantly correlated not only with the tumor extent but also with hearing impairment, contrary to the conclusions of previous reports.

Although an increase in perilymph protein in the affected cochlea is well-established in patients with ANs, the exact pathophysiologic mechanism remains unknown. ${ }^{7,10-12}$ The most plausible mechanisms include blockage of the neuroaxonal transport of proteins caused by compression of the vestibulocochlear nerve, cochlear membrane damage caused by arterial stasis and a consequent increased permeability, and a cell-mediated immune reaction to the inner ear caused by the antigenic properties of AN. ${ }^{7-12,23}$ Among the 3 hypotheses, protein blockage from compression of the corresponding nerve is considered to be the dominant mechanism, with the others being minor in their contribution. Given that the cochlear signal reflecting the protein concentration correlates well with the degree of hearing loss represented by PTA in $\mathrm{AN}_{\mathrm{IAC}}$ but not in $\mathrm{AN}_{\mathrm{CPA}}$, we could hypothesize that the degree of nerve compression is a major contribution to increasing the protein concentration while the tumor is confined to the internal auditory canal. However, the compression effect on nerve bundles would not be proportional to tumor growth when the tumor begins to grow into or originate within the CPA cistern because the CPA cistern is a relatively open space as opposed to the internal auditory canal. This problem should be investigated further with future studies.

The results of our study could be important when following patients before definitive treatment. Many patients are followed with MR imaging examinations before surgery or radiation therapy to see if the tumor is growing. The cochlear signal on FLAIR images may be an additional parameter to use when monitoring 
the degree of functional impairment during the follow-up of patients with small ANs confined to the internal auditory canals.

Finally, we selected the brain stem as a reference when calculating the rSI of the cochlea instead of the contralateral cochlea because we believed that the baseline audiometric finding of the contralateral cochlea could vary from individual to individual; this difference might affect the cochlear signal on the 3D FLAIR images and, thus, the fidelity of the method. In contrast, it is easy to rule out any abnormality of the brain stem with both MR imaging and clinical history, and no patient had a brain stem abnormality in our current series. Therefore, we selected the brain stem to calculate the rSI of the cochlea instead of the contralateral cochlea.

Our study has several limitations. Because of its retrospective nature, there may be potential biases related to the heterogeneous participants and the various time intervals between the PTA and MR imaging examinations. However, we strictly applied exclusion criteria and enrolled a large number of patients within a relatively short time interval to minimize any possible influence related to this limitation. Second, to measure cochlear signal intensity, we imported image data to ImageJ and used the Wand tool, which uses a threshold to determine the boundary of the small cochlea showing signal alteration. Although this is a reproducible and reliable method compared with the placement of a hand-drawn region of interest on the small cochlea, it may inaccurately define the cochlear boundaries when the cochlea shows a subtle or no signal increase. To overcome this limitation, we created 3D T2-weighted MR images with the same section thickness as a reference and copied and pasted the ROIs drawn on the 3D T2-weighted MR images onto the same 3D FLAIR images. The interobserver agreement, which was almost perfect, also validated the reliability of our analytic method. Finally, we did not attempt to correlate tumor volume with the cochlear signal intensities on 3D FLAIR images or the results of PTA, especially in patients with tumors confined to the IAC. We believe that further studies could clarify the relationship between them with a larger number of patients with $\mathrm{AN}_{\mathrm{IAC}}$ in the near future.

\section{CONCLUSIONS}

The increased cochlear signal on 3D FLAIR images was proved to correlate with the degree of hearing impairment measured by PTA for small tumors confined to the internal auditory canal. The cochlear signal on FLAIR images may be an additional parameter to use when monitoring the degree of functional impairment during the follow-up of patients with small ANs confined to the internal auditory canals.

\section{ACKNOWLEDGMENTS}

The authors gratefully acknowledge the technical support received from Biomedical Imaging Infrastructure, Department of Radiology, Asan Medical Center.

\section{REFERENCES}

1. Bhadelia RA, Tedesco KL, Hwang S, et al. Increased cochlear fluidattenuated inversion recovery signal in patients with vestibular schwannoma. AJNR Am J Neuroradiol 2008;29:720-23

2. Lee IH, Kim HJ, Chung WH, et al. Signal intensity change of the labyrinth in patients with surgically confirmed or radiologically diagnosed vestibular schwannoma on isotropic 3D fluid-attenuated inversion recovery MR imaging at 3 T. Eur Radiol 2010;20:949-57

3. Sugiura M, Naganawa S, Nakata S, et al. 3D-FLAIR MRI findings in a patient with Ramsay Hunt syndrome. Acta Otolaryngol 2007;127:547-49
4. Sugiura M, Naganawa S, Sone M, et al. Three-dimensional fluidattenuated inversion recovery magnetic resonance imaging findings in a patient with cochlear otosclerosis. Auris Nasus Larynx 2008;35:269-72

5. Yamazaki M, Naganawa S, Kawai H, et al. Increased signal intensity of the cochlea on pre- and post-contrast enhanced 3D-FLAIR in patients with vestibular schwannoma. Neuroradiology 2009;51:855-63

6. Yoshida T, Sugiura M, Naganawa S, et al. Three-dimensional fluidattenuated inversion recovery magnetic resonance imaging findings and prognosis in sudden sensorineural hearing loss. Laryngoscope 2008; 118:1433-37

7. O'Connor AF, France MW, Morrison AW. Perilymph total protein levels associated with cerebellopontine angle lesions. Am J Otol 1981;2:193-95

8. Palva T, Raunio V. Cerebrospinal fluid and acoustic neurinoma specific proteins in perilymph. Acta Otolaryngol 1982;93:201-03

9. Rasmussen N, Bendtzen K, Thomsen J, Tos M. Antigenicity and protein content of perilymph in acoustic neuroma patients. Acta Otolaryngol 1984;97:502-08

10. Silverstein H. Labyrinthine tap as a diagnostic test for acoustic neurinoma. Otolaryngol Clin North Am 1973;6:229-44

11. Silverstein $H, S$ Schuknecht HF. Biochemical studies of inner ear fluid in man: changes in otosclerosis, Meniere's disease, and acoustic neuroma. Arch Otolaryngol 1966;84:395-402

12. Thomsen J, Saxtrup O, Tos M. Quantitated determination of proteins in perilymph in patients with acoustic neuromas. ORL J Otorhinolaryngol Relat Spec 1982;44:61-65

13. Busse RF, Hariharan H, Vu A, et al. Fast spin echo sequences with very long echo trains: design of variable refocusing flip angle schedules and generation of clinical T2 contrast. Magn Reson Med 2006;55:1030-37

14. Chagla GH, Busse RF, Sydnor R, et al. Three-dimensional fluid attenuated inversion recovery imaging with isotropic resolution and nonselective adiabatic inversion provides improved three-dimensional visualization and cerebrospinal fluid suppression compared to two-dimensional flair at 3 Tesla. Invest Radiol 2008;43:547-51

15. Kallmes DF, Hui FK, Mugler JP. Suppression of cerebrospinal fluid and blood flow artifacts in FLAIR MR imaging with a single-slab three-dimensional pulse sequence: initial experience. Radiology 2001;221:251-55

16. Melhem ER, Jara H, Eustace S. Fluid-attenuated inversion recovery MR imaging: identification of protein concentration thresholds for CSF hyperintensity. AJR Am J Roentgenol 1997;169:859-62

17. Mugler JP, Bao S, Mulkern RV, et al. Optimized single-slab threedimensional spin-echo MR imaging of the brain. Radiology 2000;216:891-99

18. Naganawa S, Koshikawa T, Nakamura T, et al. Comparison of flow artifacts between 2D-FLAIR and 3D-FLAIR sequences at $3 \mathrm{~T}$. Eur Radiol 2004;14:1901-08

19. Otake H, Sugiura M, Naganawa S, et al. 3D-FLAIR magnetic resonance imaging in the evaluation of mumps deafness. Int J Pediatr Otorhinolaryngol 2006;70:2115-17

20. Sugiura M, Naganawa $S$, Sato E, et al. Visualization of a high protein concentration in the cochlea of a patient with a large endolymphatic duct and sac, using three-dimensional fluid-attenuated inversion recovery magnetic resonance imaging. J Laryngol Otol 2006;120:1084-86

21. Sugiura M, Naganawa $S$, Teranishi M, et al. Inner ear hemorrhage in systemic lupus erythematosus. Laryngoscope 2006;116:826-28

22. Committee on Hearing and Equilibrium guidelines for the evaluation of hearing preservation in acoustic neuroma (vestibular schwannoma): American Academy of Otolaryngology-Head and Neck Surgery Foundation, INC. Otolaryngol Head Neck Surg 1995;113:179-80

23. O'Connor AF, Luxon LM, Shortman RC, et al. Electrophoretic separation and identification of perilymph proteins in cases of acoustic neuroma. Acta Otolaryngol 1982;93:195-200 